\title{
Modulation of the Functions of Goat Peripheral Blood Mononuclear Cells by Fasciola gigantica Thioredoxin Peroxidase In Vitro
}

\author{
Ai-Ling Tian ${ }^{1,+}{ }^{\dagger}$, Xiaowei Tian ${ }^{2,+}{ }^{\dagger}$, Dan Chen ${ }^{1}$, Mingmin Lu ${ }^{2}$, Guillermo Calderón-Mantilla ${ }^{3}$, \\ Xiao-Dan Yuan ${ }^{1}$, Xiangrui Li ${ }^{2}\left(\mathbb{D}\right.$, Hany M. Elsheikha ${ }^{4, *} \mathbb{D}$ and Xing-Quan Zhu ${ }^{1,5, * \mathbb{D}}$ \\ 1 State Key Laboratory of Veterinary Etiological Biology, Key Laboratory of Veterinary Parasitology of Gansu \\ Province, Lanzhou Veterinary Research Institute, Chinese Academy of Agricultural Sciences, \\ Lanzhou 730046, China; ailingtian@hotmail.com (A.-L.T.); chendan0623@hotmail.com (D.C.); \\ yuanxiaodan0118@outlook.com (X.-D.Y.) \\ 2 College of Veterinary Medicine, Nanjing Agricultural University, Nanjing 210095, China; \\ tianxw2020@163.com (X.T.); lumingmin2008@hotmail.com (M.L.); lixiangrui@njau.edu.cn (X.L.) \\ 3 Facultad de Ingeniería, Universidad de La Sabana, Campus del Puente del Común, Km. 7, Autopista Norte \\ de Bogotá. Chía, Cundinamarca 140013,Colombia; guillermo.calderon@unisabana.edu.co \\ 4 Faculty of Medicine and Health Sciences, School of Veterinary Medicine and Science, University of \\ Nottingham, Sutton Bonington Campus, Loughborough LE12 5RD, UK \\ 5 College of Veterinary Medicine, Shanxi Agricultural University, Jinzhong 030801, China \\ * Correspondence: hany.elsheikha@nottingham.ac.uk (H.M.E.); xingquanzhu1@hotmail.com (X.-Q.Z.) \\ + These two authors contributed equally to this work.
}

Received: 3 August 2020; Accepted: 10 September 2020; Published: 17 September 2020

\begin{abstract}
The liver fluke Fasciola gigantica has a remarkable ability to establish a long-term infection within the hepatobiliary system of the mammalian definitive host. F. gigantica achieves this by producing excretory-secretory molecules, which have immunomodulatory activities. In an effort to elucidate the immunomodulatory functions of $F$. gigantica thioredoxin peroxidase protein (FgTPx), we expressed recombinant FgTPx (rFgTPx) in Escherichia coli bacteria and examined its effects on several functions of goat peripheral blood mononuclear cells (PBMCs) in vitro. Sequence analysis revealed that FgTPx is related to a thioredoxin-like superfamily. Western blot analysis showed that rFgTPx was recognized by the sera of goats experimentally infected by F. gigantica. The specific binding of rFgTPx protein to the surface of goat PBMCs was demonstrated by immunofluorescence staining. We investigated the influence of serial concentrations of $\mathrm{rFgTPx}$ on various functions of goat PBMCs. All concentrations of rFgTPx increased the secretion of interleukin-2 (IL-2), IL-4, IL-10, IL-17, transforming growth factor-beta (TGF- $\beta$ ), and interferon gamma (IFN- $\gamma$ ), but inhibited PBMC proliferation, migration, and monocyte phagocytosis. Goat PBMCs exposed to $20-40 \mu \mathrm{g} / \mathrm{mL}$ of $\mathrm{rFgTPx}$ secreted increased levels of nitric oxide (NO), and $10-40 \mu \mathrm{g} / \mathrm{mL}$ of $\mathrm{rFgTPx}$ promoted cell apoptosis. These findings indicate that $\mathrm{rFgTPx}$ influences various functions of goat PBMCs by interacting with a large number of cellular targets, ultimately to promote the parasite's survival. The roles of rFgTPx and their interacting proteins warrant further investigation.
\end{abstract}

Keywords: Fasciola gigantica; thioredoxin peroxidase; immunoregulation; peripheral blood mononuclear cells; immune responses

\section{Introduction}

Fasciolosis is a zoonotic parasitic disease caused by the liver flukes Fasciola gigantica and Fasciola hepatica [1]. The estimated economic loss every year due to fasciolosis is over 3 billion US dollars 
worldwide [2]. Also, fasciolosis has been listed by the World Health Organization (WHO) as one of the neglected tropical human diseases [3-5]: at least 240 million people worldwide are readily infected, and 180 million people are at risk of infection [6]. Given this serious impact on both the human health and sustainable agricultural economy, and because of the increase of anthelmintic resistance, alternative control strategies, such as vaccination and understanding the genetic basis of the host's innate resistance, have received increasing attention [7-9]. Some immunoprotective antigens have been identified, such as the immunoglobulin $G$ subclass and fatty acid binding protein [10-13]. Despite these efforts, no commercial vaccine is available as yet. More understanding of the molecular mechanism of F. gigantica infection can provide new insight into the protective immune responses, which ultimately can facilitate the development of new immunoregulatory therapeutic interventions.

Liver flukes of the genus Fasciola generally cause chronic (long-term) infections and survive well inside their host, which has been attributed to the parasite's ability to evade immune defense mechanisms [14-16]. Excretory and secretory products (ESPs), containing many active molecules, have been produced by these parasites under in vitro and in vivo conditions [17]. Previous reports showed that different molecules of ESPs from F. gigantica (FgESPs) can cause various immune responses-for example, some molecules can simulate host defense against parasites, while other have exerted the opposite effect [16,18-21]. Of particular importance to their survival is the ability of liver flukes to employ an anti-oxidant, redox-based system that protects the liver flukes against the reactive oxygen and nitrogen species produced by host immune cells and endogenous cellular metabolism [22]. Thioredoxin (FhTRx) and the related protein peroxiredoxin (FhPRx), key components of this anti-oxidant system, were amongst the most abundant proteins detected in the secretome of newly excysted juveniles (NEJ) of F. hepatica [23].

Our preliminary proteomic studies have indicated the potential immunomodulatory roles of thioredoxin peroxidase (TPx), one of the ESPs of F. gigantica, by revealing its ability to bind to peripheral blood mononuclear cells (PBMCs) isolated from infected goats (unpublished data). However, the effect of F. gigantica TPx (FgTPx) on the functions of goat PBMCs is unknown. In the present study, we cloned and expressed the FgTPx gene, and explored the outcomes of exposing goat PBMCs to serial concentrations of recombinant FgTPx ( $\mathrm{rFgTPx}$ ) on various functions of goat PBMCs in vitro. Our data revealed diverse effects of $\mathrm{rFgTPx}$ on the functions of goat PBMCs, which seem to promote the parasite's survival and establishment of a persistent infection. These results present rFgTPx as a new potential target for the development of immunomodulatory interventions for the control of F. gigantica infection.

\section{Results}

\subsection{De Novo Prediction of FgTPx Protein}

Protein blast results showed that the FgTPx protein is homologous to human peroxiredoxin-2 (PRDX2), with an identity of $62 \%$, an E-value of 1e-75, a query cover of $78 \%$, and a max score of 229. The human PRDX2 protein is a 197-amino-acid protein, with a molecular mass of $\sim 21.9 \mathrm{KDa}$ (Uniprot ID: P32119). Rosetta-based prediction was used to generate a de novo model of FgTPx protein (Figure 1). 


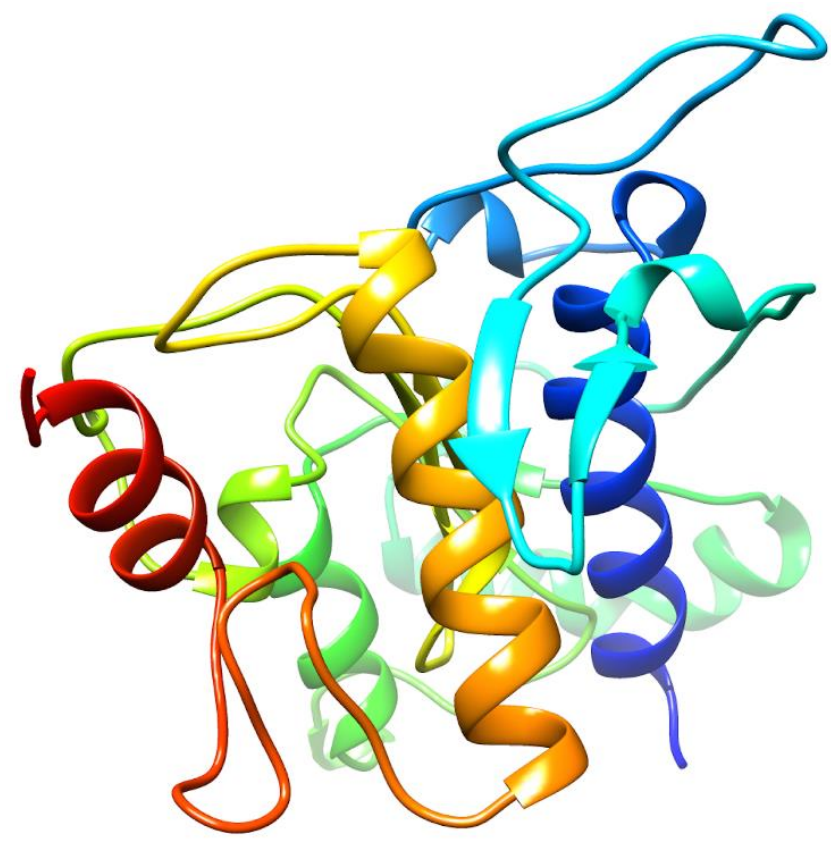

Figure 1. De novo three-dimensional (3D) model of Fasciola gigantica thioredoxin peroxidase (FgTPx) protein, constructed based on the ab initio method of protein modeling using Rosetta.

\subsection{Cloning and Sequence Analysis of FgTPx}

The PCR product corresponding to the FgTPx gene ( 711 bp) was obtained and successfully cloned into a pMD19-T cloning vector. Nucleic acid sequencing showed that the positive clones of pMD19-T/FgTP $x$ contained an insert of $711 \mathrm{bp}$. The sequence of $F g T P x$ gene encodes a protein of 236 amino acids, with a molecular mass of $26.57 \mathrm{kDa}$ and a theoretical isoelectric point (pI) of 8.01. Conserved domains analysis showed that the protein belonged to a thioredoxin-like super family. Amino acid sequence alignment of $F g T P x$ showed $100 \%$ homology with the corresponding region of F. gigantica sequence available in the National Center for Biotechnology Information (NCBI) database (GenBank accession no. ABY85785.1).

\subsection{Expression, Purification, and Detection of $r F g T P x$}

The fragment of FgTPx gene was successfully cloned into pET-28a(+) vectors, and the positive clones were designated as pET-28a/FgTPx. Protein expression was induced by isopropyl- $\beta-\mathrm{D}-$ thiogalactopyranoside (IPTG) in an E. coli BL21 (DE3) strain. The recombinant protein (rFgTPx) was expressed as His-tagged fusion proteins that were mainly produced as inclusion bodies (Figure 2). After purification, the concentrated protein was detected with a molecular mass $\sim 26 \mathrm{kDa}$ on the SDS-PAGE gel (Figure 3A). Western blot analysis showed that rFgTPx migrated at about $26 \mathrm{kDa}$ and could be recognized by anti-F. gigantica serum from experimentally infected goats, but did not react with the serum of an uninfected goat (Figure 3B). The rFgTPx was also detected using rabbit serum containing specific anti-rFgTPx antibodies, but was not reactive to naïve rabbit serum (Figure 3C). 


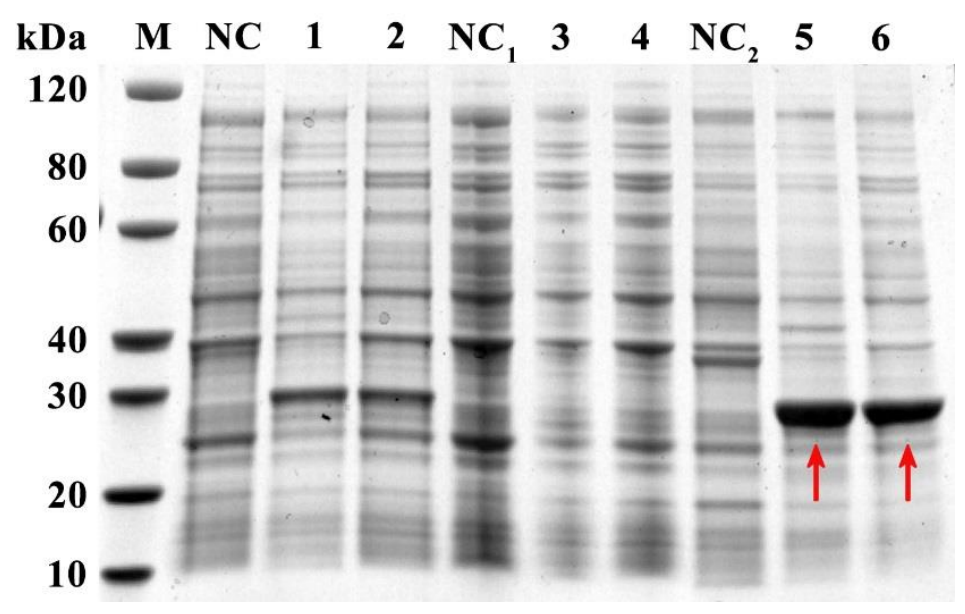

Figure 2. SDS-PAGE analysis of recombinant FgTPx (rFgTPx). Lane M: protein molecular weight marker in $\mathrm{kDa}$; Lane NC: bacterial cell lysate without induction; Lane 1: cell lysate with induction for $16 \mathrm{~h}$ at $15{ }^{\circ} \mathrm{C}$; Lane 2: cell lysate with induction for $4 \mathrm{~h}$ at $37^{\circ} \mathrm{C}$; Lane $\mathrm{NC1}$ : supernatant of cell lysate without induction; Lane 3: supernatant of cell lysate with induction for $16 \mathrm{~h}$ at $15^{\circ} \mathrm{C}$; Lane 4: supernatant of cell lysate with induction for $4 \mathrm{~h}$ at $37^{\circ} \mathrm{C}$; Lane NC2: pellet of cell lysate without induction; Lane 5: pellet of cell lysate with induction for $16 \mathrm{~h}$ at $15^{\circ} \mathrm{C}$; Lane 6: pellet of cell lysate with induction for $4 \mathrm{~h}$ at $37^{\circ} \mathrm{C}$. The red arrows denote the recombinant protein ( $\left.\mathrm{rFgTPx}\right)$.
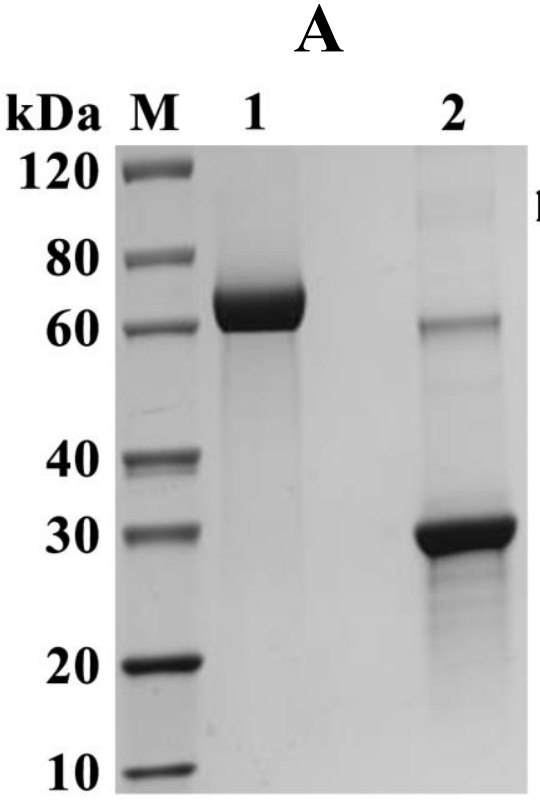

B

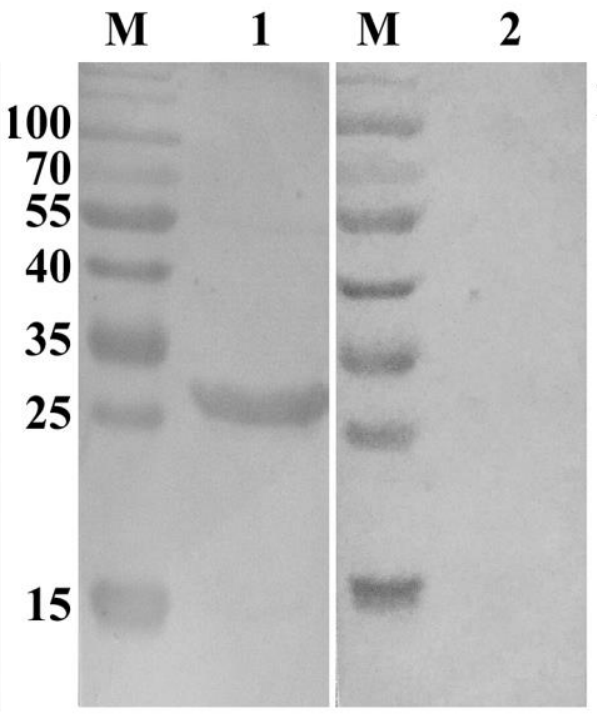

C

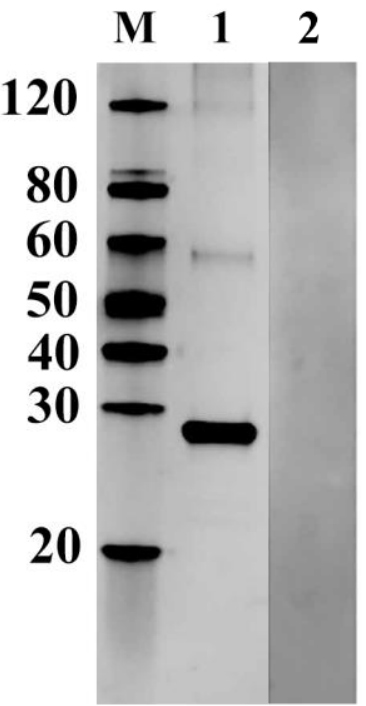

Figure 3. SDS-PAGE and Western-blot analyses of the purified recombinant protein, and rFgTPx from the sonicated pellet of E. coli. (A) Proteins were resolved on $12 \%$ acrylamide gels and stained with Coomassie brilliant blue R250. Lane M: protein molecular weight marker in kDa; Lane 1: $5 \mu \mathrm{g}$ bovine serum albumin; Lane 2: purified rFgTPx appeared as a single band of $\sim 26 \mathrm{kDa}$. (B,C) The protein of interest was run under non-reducing conditions and visualized using a chemiluminescent horseradish peroxidase substrate. Lane M: protein molecular weight marker in $\mathrm{kDa}$. (B) Lane 1 was loaded with rFgTPx. Serum from F. gigantica-infected goats detected a single band of $\sim 26 \mathrm{kDa}$; Lane 2 was loaded with rFgTPx that did not react with serum of uninfected goat. (C) Lane 1 was loaded with rFgTPx and incubated with rabbit serum containing specific anti-rFgTPx antibodies, showing a single band at $\sim 26 \mathrm{kDa}$ band; Lane 2 was loaded with rFgTPx that did not react with naïve rabbit serum. 


\subsection{Immunofluorescence Assay}

Cultured goat PBMCs incubated with rFgTPx were examined by immunofluorescence assay (IFA) using confocal microscopy (40× magnification). As shown in Figure 4, in the presence of the recombinant protein, the rabbit-derived anti-rFgTPx antibodies stained the PBMCs. On the contrary, no fluorescence was detected in non-treated control cells. The dense concentration of red color around PBMCs suggests that rFgTPx binds to the cell surface.

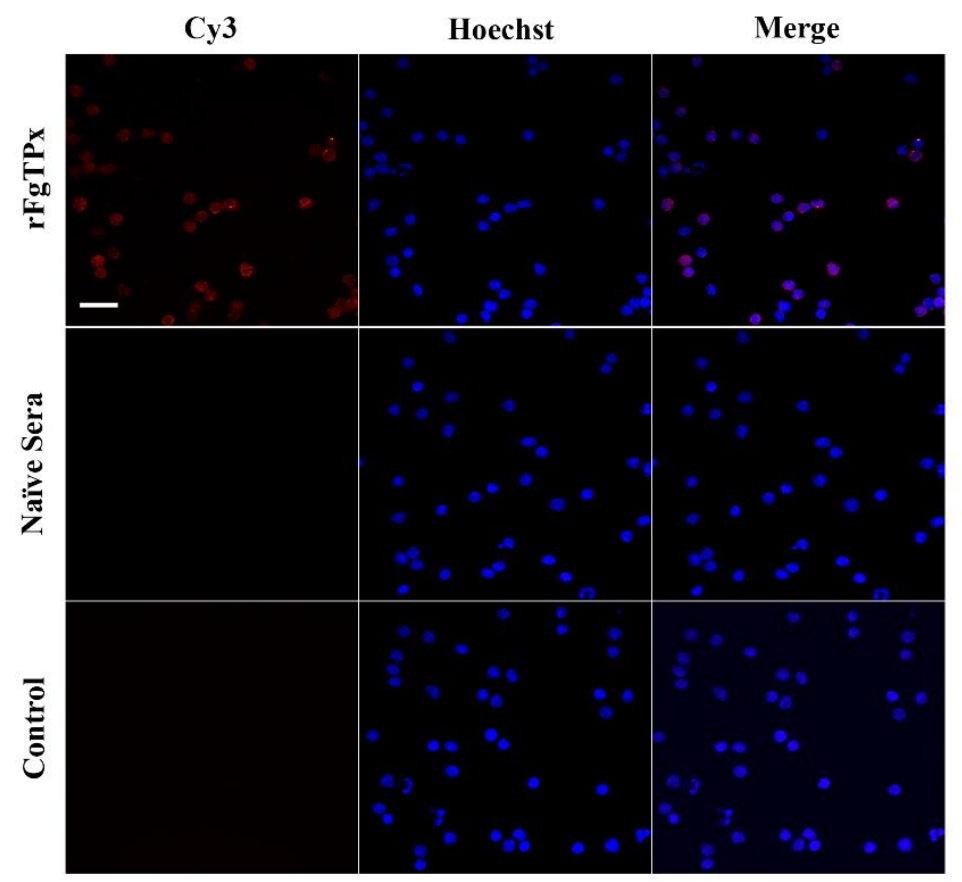

Figure 4. Fasciola gigantica-derived rFgTPx binds to the surface of goat PBMCs. Visualization of rFgTPx attachment to PBMC surfaces was carried out by incubation of PBMCs treated or untreated with rFgTPx with rabbit anti-rFgTPx primary antibody. Hoechst (blue) and Cy3-conjugated secondary antibody (red) were used to stain host cell nuclei and rFgTPx, respectively. Positive staining of cell surface was detected in rFgTPx-treated cells only. No staining was detectable in control, untreated cells. Scale bars $=10 \mu \mathrm{m}$.

\subsection{Cytokine Production, Cell Proliferation, Migration, and Production of NO}

We investigated the potential modulatory effect of rFgTPx on PBMCs. As shown in Figure 5, when cells were treated with $\mathrm{rFgTPx}(5,10,20$, and $40 \mu \mathrm{g} / \mathrm{mL})$, the production of IL-2, IL-4, IL-10, IL-17, IFN- $\gamma$, and TGF- $\beta$ were significantly increased compared to the control, untreated cells. Cell proliferation was significantly inhibited in cells treated with $\mathrm{rFgTPx}$ in a dose-dependent manner (Figure 6). All concentrations $-5 \mu \mathrm{g} / \mathrm{mL}, 10 \mu \mathrm{g} / \mathrm{mL}, 20 \mu \mathrm{g} / \mathrm{mL}$, and $40 \mu \mathrm{g} / \mathrm{mL}$-of rFgTPx significantly suppressed the migration of treated cells in a dose-dependent manner, compared with the control cells (Figure 7). As showed in Figure 8, total NO release from the cells was significantly increased in the presence of $\mathrm{rFgTPx}$ at $20 \mu \mathrm{g} / \mathrm{mL}$ and $40 \mu \mathrm{g} / \mathrm{mL}$, compared to the control, but not at $5 \mu \mathrm{g} / \mathrm{mL}$ and $10 \mu \mathrm{g} / \mathrm{mL}$. 

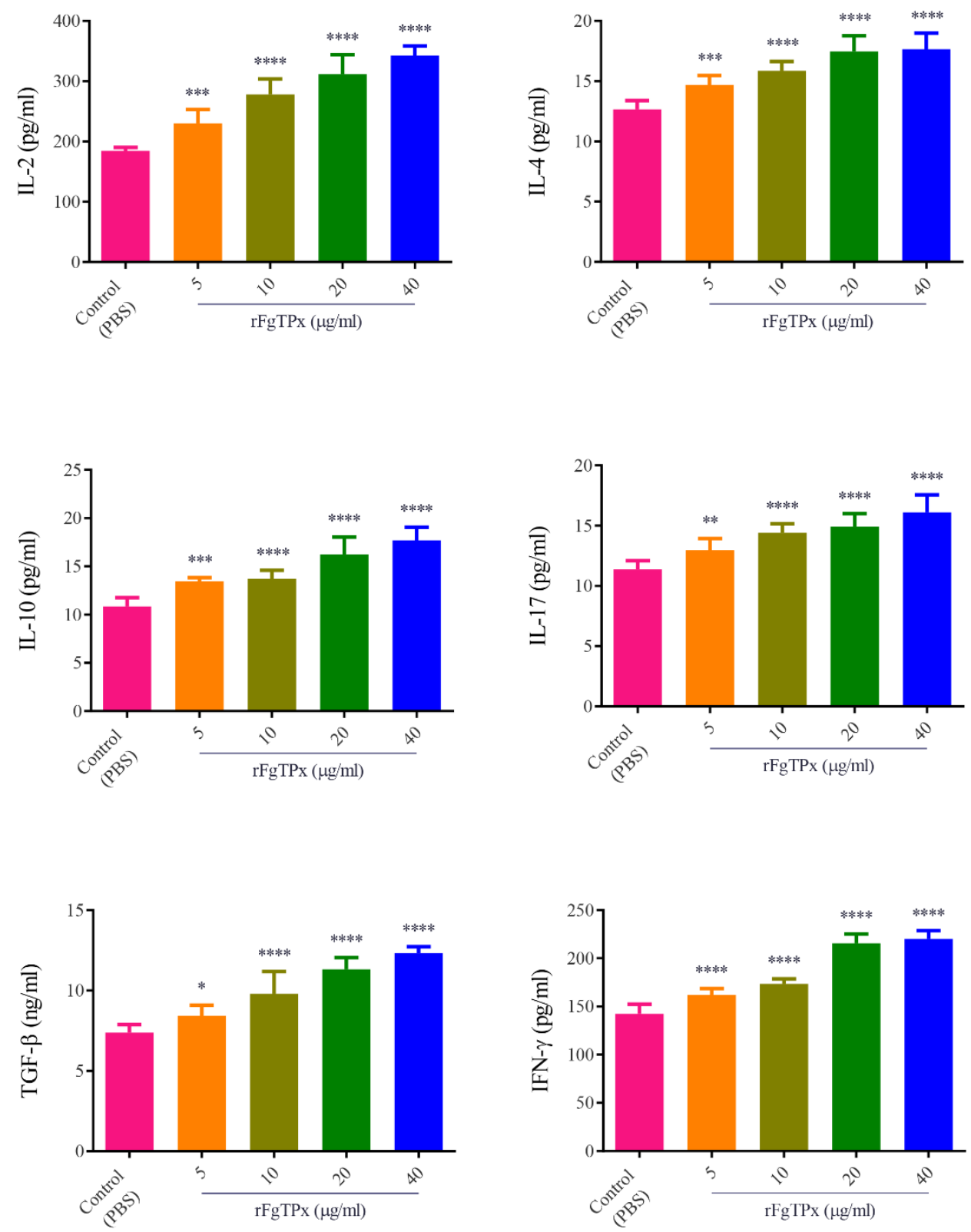

Figure 5. The rFgTPx induced polarized patterns of cytokine secretion. Goat PBMCs were incubated for $72 \mathrm{~h}$ in the presence or absence of the indicated concentrations of $\mathrm{rFgTPx}(\mu \mathrm{g} / \mathrm{mL})$. The levels of cytokine concentration in the supernatant of cultured PBMCs was quantified by ELISA. Graphs represent means \pm standard deviations of the results from three independent experiments. Asterisks indicate statistical significance between treated and untreated control goat PBMCs $\left({ }^{*} p<0.05\right.$; ${ }^{* *} p<0.01 ;{ }^{* * *} p<0.001$; $* * * * p<0.0001$ ). 


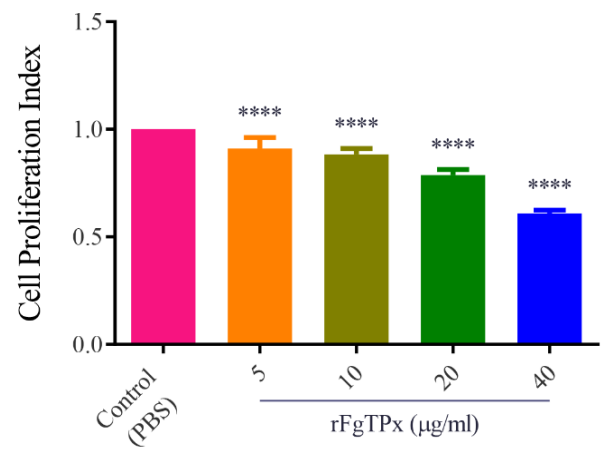

Figure 6. The rFgTPx inhibits goat PBMC proliferation. Goat PBMCs were sham-treated with phosphate-buffered saline (PBS) or with the indicated concentrations of $\mathrm{rFgTPx}(\mu \mathrm{g} / \mathrm{mL})$ and incubated for $48 \mathrm{~h}$ at $37^{\circ} \mathrm{C}$. Proliferation of cells was determined using the cell counting Kit-8 (CCK-8) assay. The rFgTPx significantly inhibited PBMC proliferation. Graphs represent means \pm standard deviations of results from three independent biological replicates. Asterisks indicate statistical significance between treated cells and control cells $\left(^{* * * *} p<0.0001\right)$.

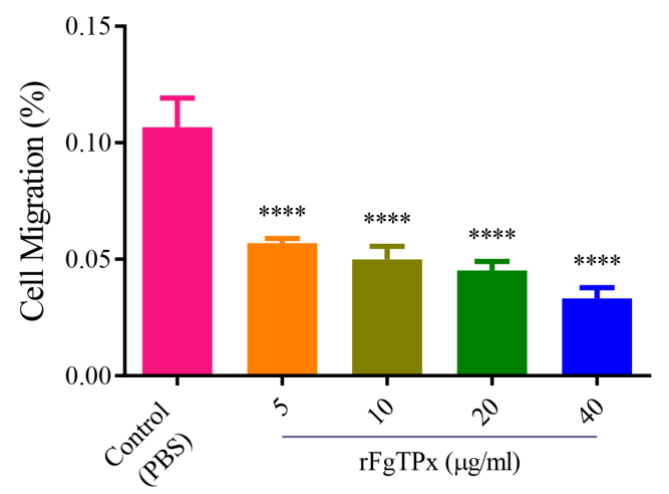

Figure 7. The rFgTPx suppressed goat PBMC migration. Goat PBMCs were sham-treated with PBS or with the shown concentrations of $\mathrm{rFgTPx}$, then cell migration percentage (\%) was determined. Graphs represent means \pm standard deviations of data from three independent experiments. The asterisks indicate significant difference between treated and sham-treated control cells $\left.{ }^{* * * *} p<0.0001\right)$.

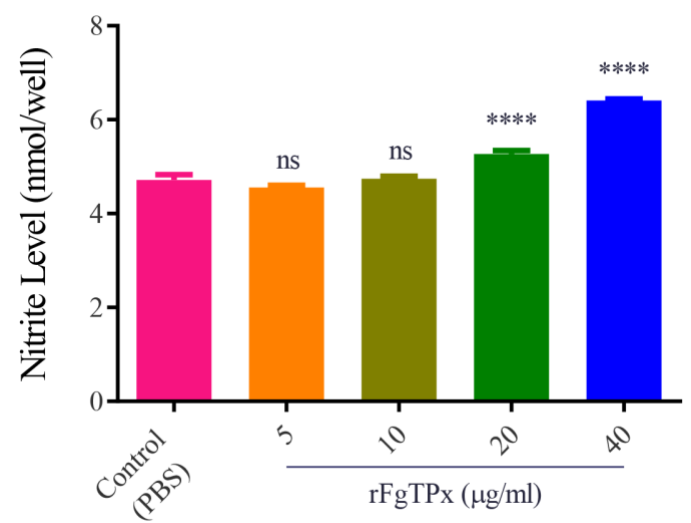

Figure 8. Effect of rFgTPx on the intracellular total NO production. Goat PBMCs were sham-treated with PBS or the indicated concentrations of rFgTPx and maintained at $37^{\circ} \mathrm{C}$. Total NO concentration produced by PBMCs was measured by Griess assay. Graphs represent means \pm standard deviations of data from three independent experiments. Asterisks indicate significant difference between treated and non-treated control cells (**** $p<0.0001$; ns: non-significant). 


\subsection{Monocyte Phagocytosis and Apoptosis}

The cell phagocytosis was examined by uptaking fluorescein isothiocyanate (FITC)-dextran. Results revealed that in $\mathrm{rFgTPx}$ induced a significant reduction in monocyte phagocytosis (Figure 9). The rFgTPx seems to have a proapoptotic effect. We detected a correlation between PBMC exposure to different concentrations of rFgTPx and the percentage of apoptotic cells. As shown in Figure 10, rFgTPx significantly induced apoptosis in goat PBMCs at $10 \mu \mathrm{g} / \mathrm{mL}, 20 \mu \mathrm{g} / \mathrm{mL}$, and $40 \mu \mathrm{g} / \mathrm{mL}$, compared to the control group, but not at $5 \mu \mathrm{g} / \mathrm{mL}$.

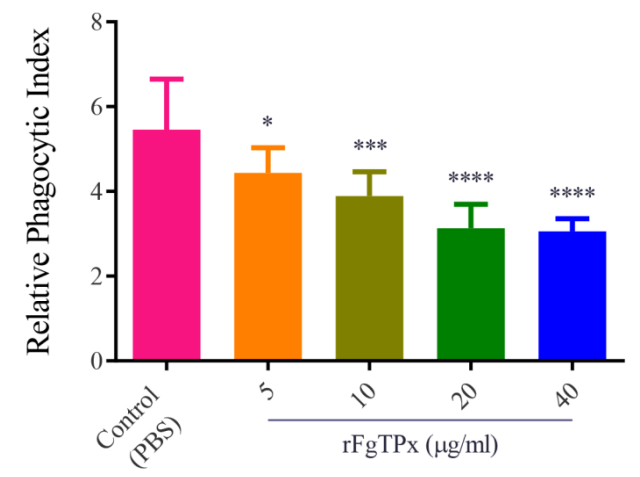

Figure 9. The rFgTPx inhibited the phagocytic ability of monocytes, as indicated by the reduction in FITC-dextran uptake in a dose-dependent manner. Graphs represent means \pm standard deviations of data from three independent experiments. Significance was set at ${ }^{*} p<0.05 ;{ }^{* * *} p<0.001$, and ${ }^{* * * *} p<0.0001$ compared to sham-treated monocytes.
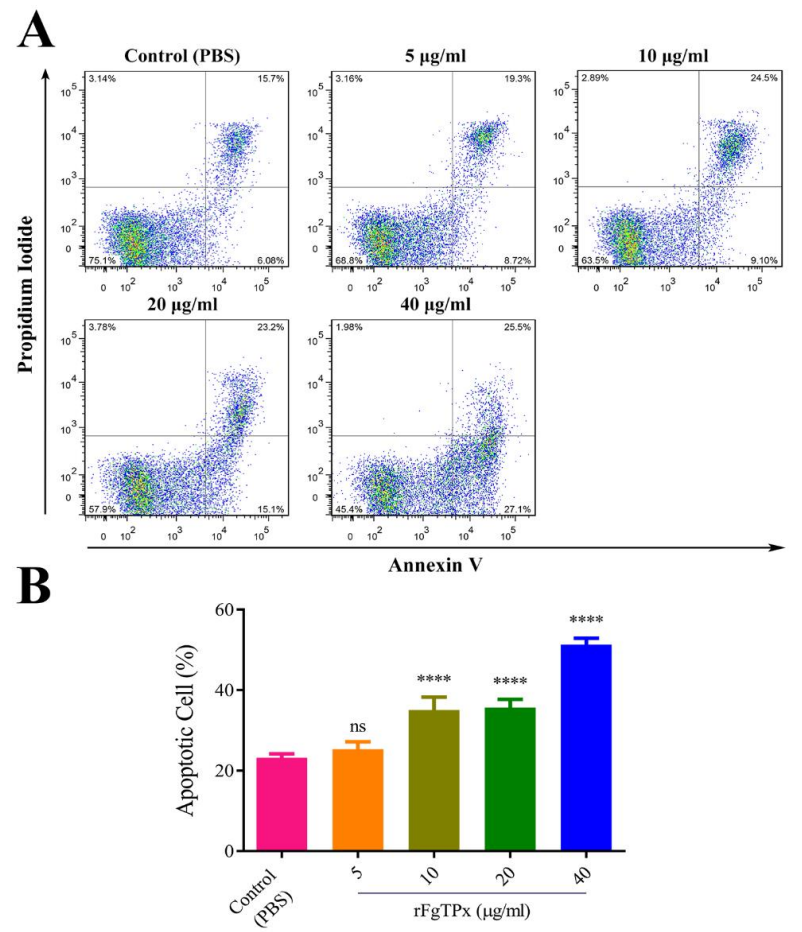

Figure 10. The rFgTPx induced apoptosis in goat PBMCs. Apoptotic cells were determined by annexin V/PI staining and flow cytometry. (A) Flow cytometric analysis shows the death of goat PBMCs in response to exposure to increasing concentrations of rFgTPx. (B) The percentage of apoptotic cells (annexin V+/PI-) compared to total cell population. Graphs represent means \pm standard deviations of data from three independent experiments. The asterisks indicate statistically significant differences between treated and untreated control PBMCs ${ }^{* * * *} p<0.0001 ;$ ns: non-significant). 


\section{Discussion}

Given our interest in understanding the immunomodulatory activities of the ESPs of the liver fluke F. gigantica, we characterized one of the F. gigantica ESPs well-known for its antioxidant properties. The thioredoxin peroxidase (TPX) gene of F. gigantica was cloned and expressed in a prokaryotic expression system. Sequence analysis and Rosetta-based prediction revealed that FgTPx is related to the thioredoxin-like superfamily, which plays a role in the antioxidant defense system of the parasite. The expressed rFgTPx protein has a molecular mass of $\sim 26 \mathrm{kDa}$, which was confirmed by Western blot analysis using sera from F. gigantica-infected goats. Using rabbit-derived, anti-rFgTPx antibody, we demonstrated the specific binding of rFgTPx to the surface of goat PBMCs. Further validation of the anti-rFgTPx antibody on F. gigantica extracts or fractions of $F$. gigantica-secreted proteins should be conducted in future studies, in order to gain more information on the physiological relevance of FgTPX binding to host cells.

It is readily known that other F. gigantica effector proteins, $\mathrm{rFg} 14-3-3 e$ [20] and $\mathrm{rFgRab} 10$ [21], alter the phagocytic function of monocytes by decreasing or increasing their phagocytic capacities, respectively, or simply kill PBMCs by inducing apoptosis. By reducing monocyte phagocytosis, rFgTPx would augment the action of rFg14-3-3e [20] and antagonize the action of rFgRab10 [21] with regard to the phagocytic capacity of monocytes. By promoting apoptosis at $10-40 \mu \mathrm{g} / \mathrm{mL}$ concentrations, $\mathrm{rFgTPx}$ seems to potentiate the action of both $\mathrm{rFg} 14-3-3 \mathrm{e}$ and $\mathrm{rFgRab} 10$ proteins. The three proteins (rFgTPx, rFg14-3-3e and $\mathrm{rFgRab} 10)$ exhibit a similar action, not only in regard to their proapoptotic activity, but also in the context of increasing NO production and reducing cell proliferation. Also, while $\mathrm{rFgTPx}$ and $\mathrm{rFg} 14-3-3$ e suppress cell migration, $\mathrm{rFgRab} 10$ seems to exert the opposite effect on cell migration.

Liver flukes have developed a variety of mechanisms to counterbalance the antiparasitic potential of the innate immune effectors of their mammalian hosts. For example, thioredoxin (TRx) protein is one of the ESPs of F. hepatica (FhPRx) that serves as a pathogen-associated molecular pattern (PAMP), increases the induction and recruitment of alternatively activated (M2) macrophages, and skews the host immunity towards a Th2 type response [24]. In our study, rFgTPx attached with high affinity to the surface of PBMCs, indicating that this protein may interact with immune cell surface receptors, thereby inducing immune response. F. gigantica is a helminth parasite that drives Th1/Th2 immune responses in its mammalian host [25-27]. F. gigantica discharges many ES molecules that seem to play roles in the induction of this type of immune response. F. gigantica-derived ESPs, identified in the present study (rFgTPx) and in previous studies (rFg14-3-3e [20] and rFgRab10 [21]), have induced Th1 (IFN- $\gamma$, IL-2) and Th2 (IL-4, IL-10, TGF- $\beta$ ) cytokines, except IL-4 and IFN- $\gamma$, whose levels were low in response to $\mathrm{rFg} 14-3-3 \mathrm{e}$. Cytokines are produced by a variety of immune cells and play various roles in protecting the host and maintaining tissue homeostasis [28,29]. In an earlier study on F. gigantica infection in buffaloes, we observed that a mixed Th1/Th2 immune response is dominant from 28 to $70 \mathrm{dpi}$, to promote F. gigantica survival while limiting host tissue damage [27].

Th1 cells mainly secrete IL- 2 and IFN- $\gamma$ to control intracellular pathogens, and IFN- $\gamma$ can activate monocytes [30], thereby promoting the release of NO [31,32]. The production of NO by a host's immune system can damage the parasite [33,34]. Th2 cells mainly secrete IL-4, which mediates humoral immunity [35] and promotes antibody-dependent killing of eosinophils, macrophages, and mast cells [36-38]. Similar to rFg14-3-3e and rFgRab10, rFgTPx promoted apoptosis in goat PBMCs. Apoptosis has been reported in sheep peritoneal leucocytes during early F. hepatica infection to improve the survival of juvenile flukes during peritoneal migration to the liver [39]. Previous research has shown that apoptotic cells may be involved in inhibiting inflammatory responses through the induction of anti-inflammatory cytokines, such as IL-10 $[40,41]$ and TGF- $\beta$ [42,43]. Hence, both IL-10 and TGF- $\beta$ cytokines are released from apoptotic goat PBMCs to counterbalance the proinflammatory response mediated by Th1 cytokines. Thioredoxin peroxidase from F. hepatica has also been shown to have antioxidant and immunosuppressive activity [44]. Our data support the hypothesis that rFgTPx modulates the immune response of PBMCs by promoting apoptosis, but further studies are needed to 
identify the involved signaling pathways and the precise molecular mechanism of PBMC apoptosis caused by rFgTPx protein. This phenomenon has also been observed in eosinophils exposed to ESPs from $F$. hepatica, suggesting that inducing apoptosis is a general mechanism used by liver flukes to manipulate and subvert the host immune system [45].

The increased level of the proinflammatory cytokine IL-17 indicates that IL-17 and Th17 contribute to immunoregulatory mechanisms during F. gigantica infection. In the course of our previous research, a modest Th2 response at the early stage of infection was found to balance harmful Th1 and Th17 responses in F. gigantica-infected buffaloes [46]. We also observed that F. gigantica infection had a significant impact on the expression of Th1, Th2, Th17, and Treg cytokines [47]. IL-17 is important in the host defense against extracellular pathogens, predominantly at mucosal sites. This cytokine can be generated by adaptive or innate immune cells and participates in host immune response against pathogens by increasing neutrophil expansion through modulating the expression of granulocyte colony-stimulating factor and by recruitment of immune cells to sites of inflammation via modulating the expression of the chemokine CXC [48,49].

Our findings suggest that while rFgTPx elicits a strong immune response and promotes the apoptosis of host cells, this protein also inhibits monocyte phagocytic function, and inhibits the proliferation and migration of PBMCs. These results suggest that rFgTPx may interfere with the differentiation and developmental pathway of PBMCs and suppresses their migration. These effects would result in a less effective immune response against $F$. gigantica by reducing the number of immune cells recruited to the infection sites, which could promote the parasite survival. Taken together, the diverse modulatory effects of rFgTPx on goat PBMCs suggest that rFgTPx protein interacts with various proteins/regulatory partners and modulates cellular processes to create niches that support the liver flukes' persistence inside the host. However, caution must be taken in the interpretation of the results obtained in the present study, because the timing of secretion and the secreted amounts of FgTPx protein in vivo are unknown. Hence, these in vitro observations may not be completely functionally relevant in the context of a real infection.

\section{Materials and Methods}

\subsection{Ethics Approval}

Experimental procedures performed in this study were reviewed and approved by the Animal Administration and Ethics Committee of Lanzhou Veterinary Research Institute, Chinese Academy of Agricultural Sciences. All animals were handled strictly according to the Animal Ethics Procedures and Guidelines of the People's Republic of China.

\subsection{Parasites and Animals}

Adult F. gigantica flukes were obtained from Guangxi Zhuang Autonomous Region (China) as described previously [20]. Eight local goats ( 3 to 6 months old) were used in this study. As previously described, goats were treated with triclabendazole to rule out Fasciola infection and kept under hygienic F. gigantica-free conditions [21]. All goats were fed with commercial hay and corn, and allowed to drink water ad libitum. Female New Zealand Rabbits (4 months old) purchased from GenScript Biotech Corp (Nanjing, China) were used to produce antibodies. Rabbits were kept in specific pathogen-free (SPR) conditions and allowed access to sterilized food and water ad libitum.

\subsection{Bioinformatis Analysis}

To predict the de novo structure of the FgTPx sequence, we identified the most homologous protein (peroxiredoxin-2 protein (PRDX2), Uniprot ID: P32119) in humans using protein BLAST [50]. Then, we performed Rosetta-based prediction to generate a de novo 3D model [51]. 


\subsection{Construction of Prokaryotic Expression Vector of FgTPX}

Total RNA extraction from adult F. gigantica was performed using a single-step protocol, as previously described [29]. The complementary DNA (cDNA) was obtained with reverse transcription polymerase chain reaction (RT-PCR), using a cDNA Kit (TaKaRa Biotechnology, Dalian, Liaoning, China), according to the manufacturer's instructions. The sequence of FgTPx gene was amplified using PCR and specific primers targeting the conserved domain sequences (CDSs) of FgTPx gene (GenBank accession no: ABY85785.1). Gene-specific primers (forward primer: 5' - CATATGCTCTCAAGTTCACTTATAATCG-3', and reverse primer: $5^{\prime}$-CTCGAGGTTGGCTGAGGAGAAATA-3') included NdeI and XhoI restriction sites (underlined). The FgTPx fragment was cloned into a pMD19-T vector (TaKaRa Biotechnology, Dalian, Liaoning, China), which was transformed into Escherichia coli DH5 $\alpha$ cells using the $\mathrm{CaCl}_{2}$ transformation method. The positive clones were verified by double digestion and sequencing. The FgTPx gene was then sub-cloned into a pET-28a(+) vector (Invitrogen, Carlsbad, CA, USA), followed by transformation into E. coli BL21 (DE3) cells using the same transformation method. The constructed recombinant plasmid was thereafter named as pET-28a/FgTPx.

\subsection{Expression and Purification of Recombinant FgTPx ( $r F g T P x)$}

The vector pET-28a encoding FgTPx were transformed to E. coli BL21 (DE3)-competent cells, and protein expression was induced with1 $\mathrm{mM}$ isopropyl- $\beta$-D-thiogalactopyranoside (IPTG; Sigma-Aldrich, USA). The purification of rFgTPx was performed as previously described. Briefly, the bacterial cells were harvested by centrifugation after $5 \mathrm{~h}$ post-IPTG induction. Following centrifugation at $10,000 \times g$, the cells were sonicated and again centrifuged, and a $\mathrm{Ni}^{2+}$-nitrilotriacetic acid (Ni-NTA) column (GE Healthcare, Chicago, IL, USA) was used to purify the rFgTPx with His-tag, according to the manufacturer's instructions. Buffer containing a linear, decreased urea gradient was used to dialyze the eluted protein, in order to be refolded and renatured. Then phosphate-buffered saline (PBS; pH 7.4) was used to exchange the urea-containing buffer. The endotoxin was removed from the protein samples using Detoxi-Gel Affinity Pak prepacked columns (Pierce Biotechnology Inc., Rockford, IL, USA). Then, the level of endotoxin of the recombinant protein ( $<1 \mathrm{EU}$ per $1 \mathrm{mg}$ ) was measured by limulus amoebocyte lysate (LAL) gel clot assay using a Pyrosate Kit (Associates of Cape Cod Inc., East Falmouth, MA, USA). The purified recombinant protein was detected by $12 \%$ SDS-PAGE, and visualized by Coomassie blue staining, and its concentration was measured by using Bradford method.

\subsection{Generation of Antibodies}

The goat anti-sera used in immunoblot analysis were obtained from F. gigantica-infected goats. Four healthy goats (4-7 months old) were infected orally with 250 viable encysted metacercariae. After three months, the goat blood was collected, and serum containing anti-F. gigantica antibodies was separated. Naïve goat sera (used as negative control) were collected from a healthy goat and stored at $-80^{\circ} \mathrm{C}$ for later use. Specific antibodies against $\mathrm{rFgTPx}$ were prepared following the protocols described previously [20], except that rabbits were used in the present study.

\subsection{Western Blot Analysis}

Western blot analysis was performed as previously described [21]. Briefly, the purified rFgTPx was loaded onto $12 \%$ SDS-PAGE gel, then transferred to Hybond-C extra nitrocellulose membranes (Amersham Biosciences, United Kingdom) and blocked with $5 \%$ bovine serum albumin (BSA) in TBS-Tween 20 (TBST) for $1 \mathrm{~h}$ at $37{ }^{\circ} \mathrm{C}$. Goat antisera (1:100 dilution with TBST containing 5\% BSA) or rabbit serum (1:200 dilution) were used as primary antibodies. Horseradish Peroxidase (HRP)-conjugated rabbit anti-goat immunoglobulin G (IgG) (Sigma, USA) (1:5000 dilution with TBST containing 5\% BSA) or goat anti-rabbit IgG-HRP antibody (Invitrogen, USA) (1:10,000 dilution) were used as secondary antibodies. Western blot assay was developed with 3,3'-diaminobenzidine (DAB; Sigma), as a chromogenic substrate, to detect the immune reaction. 


\subsection{Isolation and Culture of Goat PBMCs and Monocytes}

Goat PBMCs were isolated from the jugular vein of three healthy goats using the standard Ficoll-hypaque (GE Healthcare, USA) and gradient centrifugation, as previously described [52]. The monocytes were isolated from the PBMCs based on their adhesion to plastic plates. Finally, PBMCs and monocytes were respectively suspended to a final density of $1 \times 10^{6}$ cells $/ \mathrm{mL}$ in Roswell Park Memorial Institute (RPMI) 1640 or Dulbecco's Modified Eagle Medium (DMEM) medium, containing $10 \%$ heat-inactivated fetal bovine serum (FBS), $100 \mathrm{U} / \mathrm{mL}$ penicillin, and 10,000 U/mL penicillin and streptomycin (Gibco, Grand Island, NY, USA). Cell viability were examined by the Trypan blue dye exclusion assay.

\subsection{Immunofluorescence Staining}

Freshly collected goat PBMCs were treated with $\mathrm{rFgTPx}$ for $1 \mathrm{~h}$ at $37^{\circ} \mathrm{C}$ in a humidified atmosphere with $5 \% \mathrm{CO}_{2}$. The cells were pelleted by centrifugation at $300 \times \mathrm{g}$ for $10 \mathrm{~min}$ and washed three times in PBS to remove unbound proteins. The goat PBMCs were fixed in $4 \%$ paraformaldehyde for $10 \mathrm{~min}$ at ambient temperature, washed three times in ice-cold PBS (5 min each), and then blocked with PBS containing 4\% BSA for $1 \mathrm{~h}$. The rFgTPx-treated or non-treated (control) goat PBMCs were incubated with primary rabbit anti-rFgTPx antibody (1:500) for $1 \mathrm{~h}$ at $37^{\circ} \mathrm{C}$. After incubation, the cells were washed three times in PBS and stained with Cy3-conjugated goat anti-rabbit IgG (1:500) (Beyotime, Haimen, Jiangsu, China). The cells were washed five times after being incubated with secondary antibody at $37^{\circ} \mathrm{C}$ for $1 \mathrm{~h}$. Hoechst 33,342 (Invitrogen, Eugene, OR, USA) was used to counterstain the cell nuclei. Immunofluorescence-labeled cell preparations were analyzed using a Zeiss laser scanning microscope (LSM710, Zeiss, Jena, Germany) with the $63 \times$ oil-immersion objective and Zeiss operating system associated with the ZEN imaging program.

\subsection{Enzyme-Linked Immunosorbent Assays}

The levels of cytokines (interleukin-2 (IL-2), IL-4, IL-10, and IL-17), transforming growth factor beta (TGF- $\beta$ ), and interferon gamma (IFN- $\gamma$ ) were measured using antibodies and standards provided in the goat enzyme-linked immunosorbent assay (ELISA) kits (Mlbio, Shanghai, China). Briefly, $100 \mu \mathrm{L}$ of RPMI 1640 medium containing goat PBMCs $\left(10^{6}\right.$ cells $\left./ \mathrm{mL}\right)$ was added into 96-well tissue culture plates $(100 \mu \mathrm{L} /$ well $)$ and exposed to serial concentrations $(5,10,20$, and $40 \mu \mathrm{g} / \mathrm{mL})$ of $\mathrm{rFgTPx}$. PBMCs treated with medium only served as controls. After incubation at $37{ }^{\circ} \mathrm{C}$ and $5 \% \mathrm{CO}_{2}$ for $72 \mathrm{~h}$, the supernatant was collected, and cytokine levels were measured using ELISA kits in accordance with the manufacturer's instructions.

\subsection{Cell Proliferation Analysis}

RPMI 1640 medium containing $10^{6}$ cells/mL were placed in a 96-well tissue culture plate (100 $\mu \mathrm{L} /$ well). The rFgTPx at 5, 10, 20, and $40 \mu \mathrm{g} / \mathrm{mL}$ concentrations was added to the respective wells, and wells containing medium only served as controls. The plates were placed in $5 \% \mathrm{CO}_{2}$ incubator for $48 \mathrm{~h}$. Then, $10 \mu \mathrm{L}$ of CCK-8 reagent (Beyotime Biotechnology, Haimen, Jiangsu, China) was added. After incubation in dark for $4 \mathrm{~h}$, the absorbance was measured at $450 \mathrm{~nm}\left(\mathrm{OD}_{450}\right)$ using a microplate reader (Bio-Rad Laboratories, Hercules, CA, USA). Cell proliferation index was calculated as follows: $\mathrm{OD}_{450}$ treated cells/OD 450 control.

\subsection{Cell Migration Assay}

Cell migration was investigated using a Millicell insert with $8 \mu \mathrm{m}$ pores (Merck Millipore, Darmstadt, Hessen, Germany). Approximately $10^{6}$ cells/well were added into a 24-well tissue culture plate and stimulated with rFgTPx at 5, 10, 20, and $40 \mu \mathrm{g} / \mathrm{mL}$ concentrations in RPMI 1640 medium in a total volume of $1 \mathrm{~mL} /$ well. After incubation at $37^{\circ} \mathrm{C}$ and $5 \% \mathrm{CO}_{2}$ for $24 \mathrm{~h}$, cells were harvested, and the cell number was adjusted to $1.5 \times 10^{6}$ cells $/ \mathrm{mL}$. Then, $3 \times 10^{5}$ cells $(200 \mu \mathrm{L})$ were added into 
the upper chamber, while the bottom chamber of the inserts was loaded with $1300 \mu \mathrm{L}$ of RPMI 1640 medium. After incubation for $2 \mathrm{~h}$, the inserts were removed, and a Neubauer counting chamber was used to count the cells, which migrated through the membrane to the bottom chamber. The non-treated cells were used as a control.

\subsection{Measurement of Total Nitric Oxide (NO) Production}

To measure the production of total NO in PBMC's supernatant, a Griess assay (Abcam, Cambridge, MA, USA) was used as previously described [21]. The rFgTPx at the same concentrations stated above was used to stimulate goat PBMCs $\left(10^{6}\right.$ cells $\left./ \mathrm{mL}\right)$ in DMEM medium. After $24 \mathrm{~h}$, the culture supernatant was collected and processed using a Griess assay kit. A microplate reader (Bio-Rad Laboratories, Hercules, CA, USA) was used to measure absorbance values at $540 \mathrm{~nm}\left(\mathrm{OD}_{540}\right)$, and then the results were expressed as micromoles per liter. The non-treated cells served as control.

\subsection{Determination of Phagocytic Activity}

The effect of rFgTPx on the phagocytic activity of PBMCs was investigated by measuring the uptake of FITC-dextran by monocytes, as previously described [21]. Briefly, $10^{6}$ monocytes were inoculated to a 24-well tissue culture plate and stimulated with $\operatorname{rFgTPx}(5,10,20$, and $40 \mu \mathrm{g} / \mathrm{mL}) \mathrm{in}$ RPMI 1640 medium for $48 \mathrm{~h}$. Then the cells were collected and re-suspended in $100 \mu \mathrm{L}$ of cold PBS, then $100 \mu \mathrm{L}$ of $1 \mathrm{mg} / \mathrm{mL}$ FITC-dextran (Sigma, St Louis, MO, USA) were added. Cold PBS containing $2 \%$ FBS was used to stop the reaction. FACS Calibur analysis (BD Biosciences, San Jose, CA, USA) and FlowJo 7.6 software (Tree Star, Ashland, OR, USA) were used to collect and analyze the data. Cell phagocytosis index was determined by median fluorescence intensity (MFI) values. The blank control was set as $100 \%$. The non-treated cells were used as the control.

\subsection{Cell Apoptosis Assay}

PBMCs $\left(10^{6}\right.$ cells/mL) treated with $\operatorname{rFgTPx}(5,10,20$, and $40 \mu \mathrm{g} / \mathrm{mL})$ were incubated at $37^{\circ} \mathrm{C}$ in a humidified atmosphere with $5 \% \mathrm{CO}_{2}$. After $24 \mathrm{~h}$, an annexin V-FITC kit (Miltenyi Biotec, Bergisch Gladbach, Nordrhein-Westfalen, Germany) was used to detect cell apoptosis. The stained cells were analyzed using flow cytometry (BD Biosciences, San Jose, CA, USA). Cells treated with PBS were used as control.

\subsection{Statistical Analysis}

Data analysis was performed using GraphPad Prism ver. 7 (GraphPad Software, San Diego, CA, USA). One-way ANOVA with a Dunnett's test was performed to analyze the difference between groups. The difference was regarded as statistically significant when the $p$-value was $<0.05$.

\section{Conclusions}

We provided the first molecular and functional characterization of FgTPx. Western blot showed that $\mathrm{rFgTPx}$ is recognized by the sera of infected goats and specific rabbit serum. When incubated with goat PBMCs, all concentrations of rFgTPx increased the secretion of IL-2, IL-4, IL-10, IL-17, TGF- $\beta$, and IFN- $\gamma$, but inhibited cell proliferation and migration, and reduced monocyte phagocytosis. Also, $20-40 \mu \mathrm{g} / \mathrm{mL}$ of $\mathrm{rFgTPx}$ increased the release of total $\mathrm{NO}$, and $10-40 \mu \mathrm{g} / \mathrm{mL}$ of $\mathrm{rFgTPx}$ promoted cell apoptosis. Our data indicate that FgTPxs contribute to the immune evasion strategy employed by F. gigantica by modulating the functions of PBMCs, which represent the first line of defense against parasite infection. By modulating and inflicting damage on the effector cells of the immune system, FgTPx may modify the host capacity to counter F. gigantica invasion. Although the precise immunological mechanism remains to be elucidated, $\mathrm{rFgTPx}$ clearly impairs several functions of goat PBMCs and the subsequent development of adaptive immunity. Given the pervasive modulatory effects that FgTPx exhibited, understanding the exact functions of this effector protein may ultimately 
lead to the development of novel immunomodulatory interventions for the treatment of $F$. gigantica infection. Future studies should investigate the effect of a combination of ESPs (rFgTPx, rFg14-3-3e, and $\mathrm{rFgRab10}$ ) on the induction of an immune response by goat PBMCs in vitro and to protective immunity in vivo.

Author Contributions: Conceptualization, X.-Q.Z., X.L., and H.M.E.; software, G.C.-M. and H.M.E.; validation, X.-Q.Z. and H.M.E.; investigation, A.-L.T. and X.T.; data curation, G.C.-M. and H.M.E.; writing-original draft preparation, A.-L.T., D.C., M.L., and X.-D.Y.; writing-review and editing, X.-Q.Z., X.L., M.L., X.-D.Y., D.C., A.-L.T., X.T., G.C.-M., and H.M.E.; visualization, A.-L.T., G.C.-M., and H.M.E.; supervision, X.-Q.Z.; project administration, X.-Q.Z. All authors have read and agreed to the published version of the manuscript.

Funding: This work was supported by the National Key Basic Research Program (973 Program) of China (Grant No. 2015CB150300) and the Agricultural Science and Technology Innovation Program (ASTIP) (Grant No. CAAS-ASTIP-2016-LVRI-03).

Conflicts of Interest: The authors declare no conflict of interest. The funders had no role in the design of the study; in the collection, analyses, or interpretation of data; in the writing of the manuscript, or in the decision to publish the results.

\section{References}

1. Alasaad, S.; Soriguer, R.C.; Abu-Madi, M.; El Behairy, A.; Jowers, M.J.; Banos, P.D.; Piriz, A.; Fickel, J.; Zhu, X.Q. A TaqMan real-time PCR-based assay for the identification of Fasciola spp. Vet. Parasitol. 2011, 179, 266-271. [CrossRef] [PubMed]

2. Piedrafita, D.; Spithill, T.W.; Smith, R.E.; Raadsma, H.W. Improving animal and human health through understanding liver fluke immunology. Parasite Immunol. 2010, 32, 572-581. [CrossRef] [PubMed]

3. World Health Organization. Report of the WHO Informal Meeting on Use of Triclabendazole in Fascioliasis Control; WHO: Geneva, Switzerland, 2006.

4. World Health Organization. The "Neglected" Neglected Worms. Action against Worms; WHO: Geneva, Switzerland, 2007.

5. World Health Organization. Accelerating Work to Overcome the Global Impact of Neglected Tropical Diseases: A Roadmap for Implementation - Executive Summary; WHO: Geneva, Switzerland, 2012.

6. Meemon, K.; Sobhon, P. Juvenile-specific cathepsin proteases in Fasciola spp.: Their characteristics and vaccine efficacies. Parasitol. Res. 2015, 114, 2807-2813. [CrossRef] [PubMed]

7. Chaudhry, U.; Redman, E.M.; Raman, M.; Gilleard, J.S. Genetic evidence for the spread of a benzimidazole resistance mutation across southern India from a single origin in the parasitic nematode Haemonchus contortus. Int. J. Parasitol. 2015, 45, 721-728. [CrossRef]

8. Kaplan, R.M.; Vidyashankar, A.N. An inconvenient truth: Global worming and anthelmintic resistance. Vet. Parasitol. 2012, 186, 70-78. [CrossRef] [PubMed]

9. MacKinnon, K.M.; Bowdridge, S.A.; Kanevsky-Mullarky, I.; Zajac, A.M.; Notter, D.R. Gene expression profiles of hair and wool sheep reveal importance of Th2 immune mechanisms for increased resistance to. J. Anim. Sci. 2015, 93, 2074-2082. [CrossRef] [PubMed]

10. Allam, G.; Bauomy, I.R.; Hemyeda, Z.M.; Sakran, T.F. Evaluation of a 14.5 kDa-Fasciola gigantica fatty acid binding protein as a diagnostic antigen for human fascioliasis. Parasitol. Res. 2012, 110, 1863-1871. [CrossRef] [PubMed]

11. Figueroa-Santiago, O.; Delgado, B.; Espino, A.M. Fasciola hepatica saposin-like protein-2-based ELISA for the serodiagnosis of chronic human fascioliasis. Diagn. Microbiol. Infect. Dis. 2011, 70, 355-361. [CrossRef]

12. Nguyen, T.G.; Le, T.H.; De, N.V.; Doan, T.T.; Dao, T.H.; Vercruysse, J.; Dorny, P. Assessment of a 27-kDa antigen in enzyme-linked immunosorbent assay for the diagnosis of fasciolosis in Vietnamese patients. Trop. Med. Int. Health 2010, 15, 462-467. [CrossRef]

13. Wongkham, C.; Tantrawatpan, C.; Intapan, P.M.; Maleewong, W.; Wongkham, S.; Nakashima, K. Evaluation of immunoglobulin $\mathrm{G}$ subclass antibodies against recombinant Fasciola gigantica cathepsin L1 in an enzyme-linked immunosorbent assay for serodiagnosis of human fasciolosis. Clin. Diagn. Lab. Immunol. 2005, 12, 1152-1156. [CrossRef] 
14. Boukli, N.M.; Delgado, B.; Ricaurte, M.; Espino, A.M. Fasciola hepatica and Schistosoma mansoni: Identification of common proteins by comparative proteomic analysis. J. Parasitol. 2011, 97, 852-861. [CrossRef]

15. Jefferies, J.R.; Campbell, A.M.; van Rossum, A.J.; Barrett, J.; Brophy, P.M. Proteomic analysis of Fasciola hepatica excretory-secretory products. Proteomics 2001, 1, 1128-1132. [CrossRef]

16. Robinson, M.W.; Dalton, J.P.; O’Brien, B.A.; Donnelly, S. Fasciola hepatica: The therapeutic potential of a worm secretome. Int. J. Parasitol. 2013, 43, 283-291. [CrossRef]

17. Robinson, M.W.; Dalton, J.P. Zoonotic helminth infections with particular emphasis on fasciolosis and other trematodiases. Philos. Trans. R. Soc. Lond. B Biol. Sci. 2009, 364, 2763-2776. [CrossRef]

18. Changklungmoa, N.; Kueakhai, P.; Apisawetakan, S.; Riengrojpitak, S.; Sobhon, P.; Chaithirayanon, K. Identification and expression of Fasciola gigantica thioredoxin. Parasitol. Res. 2014, 113, 2335-2343. [CrossRef]

19. Preyavichyapugdee, N.; Sahaphong, S.; Riengrojpitak, S.; Grams, R.; Viyanant, V.; Sobhon, P. Fasciola gigantica and Schistosoma mansoni: Vaccine potential of recombinant glutathione S-transferase (rFgGST26) against infections in mice. Exp. Parasitol. 2008, 119, 229-237. [CrossRef]

20. Tian, A.L.; Lu, M.; Calderon-Mantilla, G.; Petsalaki, E.; Dottorini, T.; Tian, X.; Wang, Y.; Huang, S.Y.; Hou, J.L.; $\mathrm{Li}, \mathrm{X}$; et al. A recombinant Fasciola gigantica 14-3-3 epsilon protein (rFg14-3-3e) modulates various functions of goat peripheral blood mononuclear cells. Parasit. Vectors 2018, 11, 152. [CrossRef]

21. Tian, A.L.; Lu, M.; Zhang, F.K.; Calderon-Mantilla, G.; Petsalaki, E.; Tian, X.; Wang, W.; Huang, S.Y.; Li, X.; Elsheikha, H.M.; et al. The pervasive effects of recombinant Fasciola gigantica Ras-related protein Rab10 on the functions of goat peripheral blood mononuclear cells. Parasit. Vectors 2018, 11, 579. [CrossRef]

22. Robinson, M.W.; Hutchinson, A.T.; Dalton, J.P.; Donnelly, S. Peroxiredoxin: A central player in immune modulation. Parasite Immunol. 2010, 32, 305-313. [CrossRef]

23. Cwiklinski, K.; Jewhurst, H.; McVeigh, P.; Barbour, T.; Maule, A.G.; Tort, J.; O’Neill, S.M.; Robinson, M.W.; Donnelly, S.; Dalton, J.P. Infection by the helminth parasite Fasciola hepatica requires rapid regulation of metabolic, virulence, and invasive factors to adjust to its mammalian host. Mol. Cell. Proteomics 2018, 17, 792-809. [CrossRef] [PubMed]

24. Donnelly, S.; Stack, C.M.; O’Neill, S.M.; Sayed, A.A.; Williams, D.L.; Dalton, J.P. Helminth 2-Cys peroxiredoxin drives Th2 responses through a mechanism involving alternatively activated macrophages. FASEB J. 2008, 22, 4022-4032. [CrossRef] [PubMed]

25. Changklungmoa, N.; Phoinok, N.; Yencham, C.; Sobhon, P.; Kueakhai, P. Vaccine potential of recombinant cathepsinL1G against Fasciola gigantica in mice. Vet. Parasitol. 2016, 226, 124-131. [CrossRef] [PubMed]

26. Kumar, N.; Raina, O.K.; Nagar, G.; Prakash, V.; Jacob, S.S. Th1 and Th2 cytokine gene expression in primary infection and vaccination against Fasciola gigantica in buffaloes by real-time PCR. Parasitol. Res. 2013, 112, 3561-3568. [CrossRef] [PubMed]

27. Zhang, F.K.; Guo, A.J.; Hou, J.L.; Sun, M.M.; Sheng, Z.A.; Zhang, X.X.; Huang, W.Y.; Elsheikha, H.M.; Zhu, X.Q. Serum levels of cytokines in water buffaloes experimentally infected with Fasciola gigantica. Vet. Parasitol. 2017, 244, 97-101. [CrossRef] [PubMed]

28. Stenken, J.A.; Poschenrieder, A.J. Bioanalytical chemistry of cytokines-a review. Anal. Chim. Acta 2015, 853, 95-115. [CrossRef] [PubMed]

29. Sun, W.; Song, X.; Yan, R.; Xu, L.; Li, X. Cloning and characterization of a selenium-independent glutathione peroxidase (HC29) from adult Haemonchus contortus. J. Vet. Sci. 2012, 13, 49-58. [CrossRef]

30. Chen, H.Y.; Weng, I.C.; Li, C.S.; Wan, L.; Liu, F.T. Examination of galectins in phagocytosis. Methods. Mol. Biol. 2015, 1207, 201-213.

31. Gazzinelli, R.T.; Oswald, I.P.; James, S.L.; Sher, A. IL-10 inhibits parasite killing and nitrogen oxide production by IFN-gamma-activated macrophages. J. Immunol. 1992, 148, 1792-1796.

32. Wandurska-Nowak, E. The role of nitric oxide (NO) in parasitic infections. Wiad Parazytol. 2004, 50, 665-678.

33. Cervi, L.; Rossi, G.; Cejas, H.; Masih, D.T. Fasciola hepatica-induced immune suppression of spleen mononuclear cell proliferation: Role of nitric oxide. Clin. Immunol. Immunopathol. 1998, 87, 145-154. [CrossRef]

34. Colasanti, M.; Gradoni, L.; Mattu, M.; Persichini, T.; Salvati, L.; Venturini, G.; Ascenzi, P. Molecular bases for the anti-parasitic effect of NO (Review). Int. J. Mol. Med. 2002, 9, 131-134. [CrossRef] [PubMed]

35. Lacroux, C.; Nguyen, T.H.; Andreoletti, O.; Prevot, F.; Grisez, C.; Bergeaud, J.P.; Gruner, L.; Brunel, J.C.; Francois, D.; Dorchies, P.; et al. Haemonchus contortus (Nematoda: Trichostrongylidae) infection in lambs elicits an unequivocal Th2 immune response. Vet. Res. 2006, 37, 607-622. [CrossRef] [PubMed] 
36. Alba-Hurtado, F.; Munoz-Guzman, M.A. Immune responses associated with resistance to haemonchosis in sheep. BioMed Res. Int. 2013, 162158. [CrossRef] [PubMed]

37. Moreau, E.; Chauvin, A. Immunity against helminths: Interactions with the host and the intercurrent infections. J. Biomed. Biotechnol. 2010, 428593. [CrossRef]

38. Schallig, H.D. Immunological responses of sheep to Haemonchus contortus. Parasitology 2000, 120, S63-S72. [CrossRef]

39. Escamilla, A.; Perez-Caballero, R.; Zafra, R.; Bautista, M.J.; Pacheco, I.L.; Ruiz, M.T.; Martinez-Cruz, M.S.; Martinez-Moreno, A.; Molina-Hernandez, V.; Perez, J. Apoptosis of peritoneal leucocytes during early stages of Fasciola hepatica infections in sheep. Vet. Parasitol. 2017, 238, 49-53. [CrossRef]

40. Bzowska, M.; Guzik, K.; Barczyk, K.; Ernst, M.; Flad, H.D.; Pryjma, J. Increased IL-10 production during spontaneous apoptosis of monocytes. Eur. J. Immunol. 2002, 32, 2011-2020. [CrossRef]

41. Voll, R.E.; Herrmann, M.; Roth, E.A.; Stach, C.; Kalden, J.R.; Girkontaite, I. Immunosuppressive effects of apoptotic cells. Nature 1997, 390, 350-351. [CrossRef]

42. Chen, W.; Frank, M.E.; Jin, W.; Wahl, S.M. TGF-beta released by apoptotic T cells contributes to an immunosuppressive milieu. Immunity 2001, 14, 715-725. [CrossRef]

43. Fadok, V.A.; Bratton, D.L.; Konowal, A.; Freed, P.W.; Westcott, J.Y.; Henson, P.M. Macrophages that have ingested apoptotic cells in vitro inhibit proinflammatory cytokine production through autocrine/paracrine mechanisms involving TGF-beta, PGE2, and PAF. J. Clin. Investig. 1998, 101, 890-898. [CrossRef]

44. Donnelly, S.; O’Neill, S.M.; Sekiya, M.; Mulcahy, G.; Dalton, J.P. Thioredoxin peroxidase secreted by Fasciola hepatica induces the alternative activation of macrophages. Infect. Immun. 2005, 73, 166-173. [CrossRef]

45. Serradell, M.C.; Guasconi, L.; Cervi, L.; Chiapello, L.S.; Masih, D.T. Excretory-secretory products from Fasciola hepatica induce eosinophil apoptosis by a caspase-dependent mechanism. Vet. Immunol. Immunopathol. 2007, 117, 197-208. [CrossRef]

46. Zhang, F.K.; Zhang, X.X.; Elsheikha, H.M.; He, J.J.; Sheng, Z.A.; Zheng, W.B.; Ma, J.G.; Huang, W.Y.; Guo, A.J.; Zhu, X.Q. Transcriptomic responses of water buffalo liver to infection with the digenetic fluke Fasciola gigantica. Parasit. Vectors 2017, 10, 56. [CrossRef] [PubMed]

47. Shi, W.; Wei, Z.Y.; Elsheikha, H.M.; Zhang, F.K.; Sheng, Z.A.; Lu, K.J.; Wang, D.Y.; Huang, W.Y.; Zhu, X.Q. Dynamic expression of cytokine and transcription factor genes during experimental Fasciola gigantica infection in buffaloes. Parasit. Vectors 2017, 10, 602. [CrossRef] [PubMed]

48. Matsuzaki, G.; Umemura, M. Interleukin-17 as an effector molecule of innate and acquired immunity against infections. Microbiol. Immunol. 2007, 51, 1139-1147. [CrossRef] [PubMed]

49. Schulz, S.M.; Kohler, G.; Holscher, C.; Iwakura, Y.; Alber, G. IL-17A is produced by Th17, gammadelta T cells and other CD4- lymphocytes during infection with Salmonella enterica serovar Enteritidis and has a mild effect in bacterial clearance. Int. Immunol. 2008, 20, 1129-1138. [CrossRef] [PubMed]

50. States, D.J.; Gish, W. Combined use of sequence similarity and codon bias for coding region identification. J. Comput. Biol. 1994, 1, 39-50. [CrossRef] [PubMed]

51. Conchúir, O.C.; Barlow, K.A.; Pache, R.A.; Ollikainen, N.; Kundert, K.; O’Meara, M.J.; Smith, C.A.; Kortemme, T. A web resource for standardized benchmark datasets, metrics, and Rosetta protocols for macromolecular modeling and design. PLoS ONE 2015, 10, e0130433. [CrossRef]

52. Paclik, D.; Werner, L.; Guckelberger, O.; Wiedenmann, B.; Sturm, A. Galectins distinctively regulate central monocyte and macrophage function. Cell Immunol. 2011, 271, 97-103. [CrossRef]

(C) 2020 by the authors. Licensee MDPI, Basel, Switzerland. This article is an open access article distributed under the terms and conditions of the Creative Commons Attribution (CC BY) license (http://creativecommons.org/licenses/by/4.0/). 